\title{
Utilização da farinha de castanha de caju na elaboração de biscoito integral tipo
}

\section{cookie}

\author{
The use of cashew nut flour in the preparation of whole wheat cookies \\ Uso de harina de anacardo en la preparación de galleta integral tipo cookies
}

Recebido: 27/04/2021 | Revisado: 10/05/2021 | Aceito: 18/05/2021 | Publicado: 04/06/2021

\author{
Ysabele Yngrydh Valente Silva \\ ORCID: https://orcid.org/0000-0002-8500-1525 \\ Instituto Federal do Ceará, Brasil \\ E-mail: ysabelevalentin@gmail.com \\ Sheyla Maria Barreto Amaral \\ ORCID: https://orcid.org/0000-0003-0041-5487 \\ Instituto Federal do Ceará, Brasil \\ E-mail: sheylaamara182@gmail.com \\ Séfura Maria Assis Moura \\ ORCID: https://orcid.org/0000-0002-7968-7874 \\ Instituto Federal do Ceará, Brasil \\ E-mail: sefura@ifce.edu.br
}

\begin{abstract}
Resumo
O presente trabalhou objetivou elaborar biscoitos integrais tipo cookie, utilizando a farinha da castanha de caju (FCC) em substituição parcial à farinha de trigo, e avaliar as características microbiológicas, centesimal e sensorial. Foram elaboradas três formulações: C0: controle, produzida sem a FCC; $\mathrm{C} 50$ e C75, com $50 \%$ e $75 \%$ de adição da FCC, respectivamente. Os biscoitos foram submetidos a análises microbiológicas, de composição centesimal, análise sensorial por escala hedônica e intenção de compra. Os resultados das análises microbiológicas asseguraram a qualidade higiênico-sanitária na elaboração dos cookies, confirmando que se encontravam aptos para o consumo, estando de acordo com a legislação. $\mathrm{Na}$ análise centesimal, as formulações $\mathrm{C} 0$ e as adicionadas da farinha da castanha de caju, não apresentaram diferenças significativas em relação ao teor de proteínas e cinzas; a C0 apresentou o maior percentual de lipídios, porém, as amostras C50 e C75 apresentaram os maiores teores de fibras. Quanto ao teste sensorial, os maiores percentuais de aprovação obtidos foram para a formulação C75, com índice de aceitabilidade (IA) em torno de $80 \%$ para todos os atributos analisados. Para os atributos textura e sabor, as amostras C50 e C75 obtiveram as maiores notas. Os resultados indicam que os cookies produzidos com adição da farinha da castanha de caju apresentam importantes benefícios nutricionais e ótima aceitabilidade, constituindo um produto com importante potencial de mercado.
\end{abstract}

Palavras-chave: Anacardium occidentale L.; Aceitação sensorial; Amêndoa da castanha de caju.

\begin{abstract}
This work aimed at elaborating whole wheat cookies by using cashew nut flour (CNF) in substitution partial of wheat flour, at evaluating the microbiological, proximate, and sensorial characteristics. Three formulations were elaborated: C0: control, produced without the CNF; C50 and C75, with 50\% and 75\% CNF addition, respectively. The cookies were subjected to microbiological analysis, proximate composition and sensory acceptance by hedonic scale. The results of the microbiological analyzes ensured the hygienic-sanitary quality in the preparation of the cookies, confirming that they were fit for consumption, in accordance with the legislation. In the centesimal analysis, the $\mathrm{C} 0$ formulations and the ones with cashew nut flour did not present significant differences in relation to the protein and ash content; C0 had the highest percentage of lipids; however, samples C50 and C75 had the highest fiber content. Concerning the sensory test, the highest approval percentages were obtained by formulation C75, with an Acceptability Index of around $80 \%$ for all the analyzed attributes. For the texture and flavor attributes, samples C50 and C75 obtained the highest grades. The results indicate that the cookies produced with the addition of the cashew nut flour have important nutritional benefits and excellent acceptability, constituting a product with important market potential.
\end{abstract}

Keywords: Anacardium occidentale L.; Sensory acceptance; Cashew chestnut almond.

\section{Resumen}

El presente trabajo tuvo como objetivo la elaboración de galletas integrales tipo cookies, utilizando la harina de anacardo (HA) en sustitución parcial de la harina de trigo, y evaluar las características microbiológicas, próximas y sensoriales. Se elaboraron tres formulaciones: C0: control, elaborada sin HA; C50 y C75, con adición de 50\% y 75\% 
de HA, respectivamente. Las galletas se sometieron a análisis microbiológico, composición próxima y aceptación sensorial por escala hedónica, intención de compra. Los resultados de los análisis microbiológicos aseguraron la calidad higiénico-sanitaria en la elaboración de las galletas tipo cookies, confirmando que eran aptas para el consumo, estando de acuerdo con la legislación. En el análisis centesimal, las formulaciones de C0 y las agregadas de la harina de anacardo, no presentaron diferencias significativas en relación al contenido de proteínas y cenizas; C0 tuvo el mayor porcentaje de lípidos, sin embargo, las muestras C50 y C75 tuvieron el mayor contenido de fibra. En cuanto a la prueba sensorial, los mayores porcentajes de aprobación obtenidos fueron para la formulación C75, con un Índice de Aceptabilidad en torno al $80 \%$ para todos los atributos analizados. Para los atributos textura y sabor, las muestras C50 y C75 obtuvieron las puntuaciones más altas. Los resultados indican que las galletas tipo cookies producidas con la adición de la harina de anacardo tienen importantes beneficios nutricionales y una excelente aceptabilidad, constituyendo un producto con importante potencial de mercado.

Palabras clave: Anacardium occidentale L.; Aceptación sensorial; Semilla de anacardo.

\section{Introdução}

O cajueiro é uma grande fonte de renda para alguns estados do Nordeste, sobretudo nas regiões semiáridas, gerando várias empresas do setor agroindustrial, principalmente de pequeno e médio porte (Instituto Brasileiro de Geografia e Estatística [IBGE], 2017). Conjuntamente, o estado do Ceará se destaca como o principal produtor da castanha de caju, o fruto verdadeiro do cajueiro (Anacardium occidentale L.), com grande importância no comércio internacional, e cuja amêndoa é o principal produto gerado (Companhia Nacional de Abastecimento [CONAB], 2017).

Com um aumento expressivo à taxa média de 13,9\% entre 2015 e 2019, e representando 62,9\% da produção nacional em 2019, de acordo com informação do IBGE, o estado do Ceará teve uma produção de castanha de caju estimada em 97,2 mil toneladas para o ano de 2020, aumento projetado de 10,9\% na comparação com o ano anterior (CONAB, 2020). E em relação a produção da castanha em casca (in natura), a estimativa para 2020 foi de 150,6 mil toneladas, com um aumento de 8,1\% em comparação com o ano anterior, quando a produção se situou em 139,3 mil toneladas (CONAB, 2020).

Segundo Paiva, Neto, Pessoa e Leite (2006), o aproveitamento integral do caju pode se dar tanto em escala industrial como em escala familiar. Desde a amêndoa da castanha do caju (ACC), em grande parte exportada para muitos países, e mais comumente consumida torrada em óleo comestível; passando por doces e compotas, pedúnculos desidratados, sucos integrais e concentrados, néctar, polpa congelada e cajuína.

Consumida globalmente por seus atributos nutricionais e sensoriais desejáveis, a ACC constitui uma boa fonte de lipídios (48,35\%), seguido das proteínas (21,76\%) e amido (17,30\%), assim como representa uma importante fonte de selênio (Chaves, Azevedo \& Oliveira, 2020; Melo, Oliveira, Feitosa, Feitosa \& Oliveira, 2017). De acordo Freitas e Naves (2010), a ACC apresenta aminoácidos essenciais que atendem a grande parte das necessidades de adultos e crianças em idade escolar.

No momento atual, apesar do grande consumo de produtos congelados, prontos e semiprontos, e da grande procura pela rede de fastfoods, há um aumento da parcela da população que vem se preocupando mais com a ingestão de alimentos saudáveis, o que torna importante a busca por elaborações de formulações com um melhor valor nutricional (Chaves et al., 2020), que segundo Gadani, Mileski, Peixoto e Agostini (2017), está relacionado aos fatores químicos, físicos e biológicos (microbiológicos e enzimáticos), desde a fabricação do produto até o consumo; acrescentando que informações sobre a estabilidade e as características das matérias-primas podem contribuir para aumentar o uso e melhorar a qualidade dos alimentos processados.

De acordo com Melo et al. (2017), e Owiredu, Laryea e Barimah (2014), a ACC tem sido utilizada na elaboração de diversos produtos de panificação. Assim como Ibrahim, Omosolis, Oloye, Aladekoyi e Ogundowole (2010), constatam em seu estudo que a farinha da amêndoa da castanha do caju (FCC) pode ser usada na panificação para melhorar a textura, consistência e aparência dos produtos, devido a propriedades como densidade aparente, capacidade de formação de espuma e estabilidade. 
Destarte, considerando que no processamento da castanha ocorre um alto índice de quebra das amêndoas, que apresentam um preço reduzido em comparação às inteiras (Lima, Pinto \& Magalhães, 2018), a elaboração de novos produtos a partir das amêndoas quebradas da castanha de caju, é uma forma de agregar valor ao subproduto, além de ser uma boa opção para a preparação de alimentos ricos nutricionalmente, ao modificar uma receita básica, incrementando-a com uma fonte de gorduras saudáveis (lipídios), carboidratos, proteínas, minerais, fibras e compostos bioativos (Owiredu et al., 2014).

Diante do exposto, o presente trabalho teve como objetivo a elaboração de biscoitos integrais tipo cookie, utilizando a farinha da amêndoa da castanha de caju (FCC), obtida de amêndoas quebradas provenientes do processamento, em diferentes proporções. Além de avaliar as características microbiológicas, físico-químicas e sensoriais dos biscoitos, comparando as formulações.

\section{Metodologia}

\subsection{Tipo de estudo}

$\mathrm{O}$ estudo refere-se a uma pesquisa de natureza experimental, desenvolvida segundo a metodologia quantitativa (Kauark, Manhães \& Medeiros, 2010).

A elaboração do produto e as análises foram realizadas no Instituto Federal de Educação, Ciência e Tecnologia do Estado do Ceará, Campus Limoeiro do Norte, entre agosto de 2018 à julho de 2019.

\subsection{Obtenção da matéria-prima}

As amêndoas da castanha de caju quebradas utilizadas neste trabalho foram adquiridas em uma empresa de beneficiamento de castanhas localizada no município de Fortaleza-CE (Latitude: 3 43' 6" Sul, Longitude: 38 32' 36" Oeste). Os demais ingredientes (farinha de trigo integral, açúcar demerara, sal, essência de baunilha, ovo, canela em pó e manteiga) foram adquiridos no comércio local da cidade de Limoeiro do Norte-CE (Latitude: 5 8' 56" Sul, Longitude: 38 5' 52" Oeste).

\subsection{Elaboração da farinha da castanha de caju}

Para elaboração da FCC foi utilizada a metodologia de Melo et al. (2017), adaptada. As amêndoas das castanhas de caju quebradas foram inicialmente selecionadas e distribuídas em bandejas de aço inoxidável, levadas ao forno convencional pré-aquecido, permanecendo por um período de 15 minutos, à $180{ }^{\circ} \mathrm{C}$. Posteriormente, o material foi processado em liquidificador doméstico e peneirado em peneira convencional utilizada em cozinha doméstica, obtendo-se a FCC. A mesma foi armazenada em recipientes de polietileno transparente com tampa, previamente sanitizados e devidamente codificados, protegidos do sol, calor e luz, a fim de preservar suas características físico-químicas e nutricionais.

\subsection{Elaboração dos cookies}

Realizou-se a elaboração das formulações, sendo C0 a formulação controle, sem a adição da FCC; C50 a formulação com 50\% da FCC (162 g) e 50\% (163 g) da farinha de trigo integral; e a formulação C75, composta por 75\% (244 g) da FCC, e $25 \%(81 \mathrm{~g})$ da farinha de trigo integral. As quantidades dos demais ingredientes foram as mesmas em todas as formulações (Tabela 1).

O processamento dos cookies (Figura 1) foi realizado em duas repetições, seguindo a metodologia de Melo et al. (2017), adaptada. 
Tabela 1. Ingredientes utilizados nas formulações dos cookies com farinha de castanha de caju.

\begin{tabular}{lccc}
\hline \multirow{2}{*}{ Ingredientes } & \multicolumn{3}{c}{ Formulação (g) } \\
\cline { 2 - 4 } & C0 & C50 & C75 \\
\hline Farinha de trigo integral & 325 & 163 & 81 \\
Farinha de castanha de caju (FCC) & 0 & 162 & 244 \\
Açúcar demerara & 200 & 200 & 200 \\
Manteiga & 200 & 200 & 200 \\
Ovo & 100 & 100 & 100 \\
Sal & 5 & 5 & 5 \\
Canela em pó & 5 & 5 & 5 \\
Essência de baunilha & 5 & 5 & 5 \\
\hline \multicolumn{1}{c}{ TOTAL } & $840 \mathrm{~g}$ \\
\hline
\end{tabular}

C0: controle; C50: 50\% de FCC; C75: 75\% de FCC. Fonte: Autores, 2020.

Figura 1. Fluxograma de processamento dos cookies elaborados com diferentes concentrações da farinha de castanha de caju.

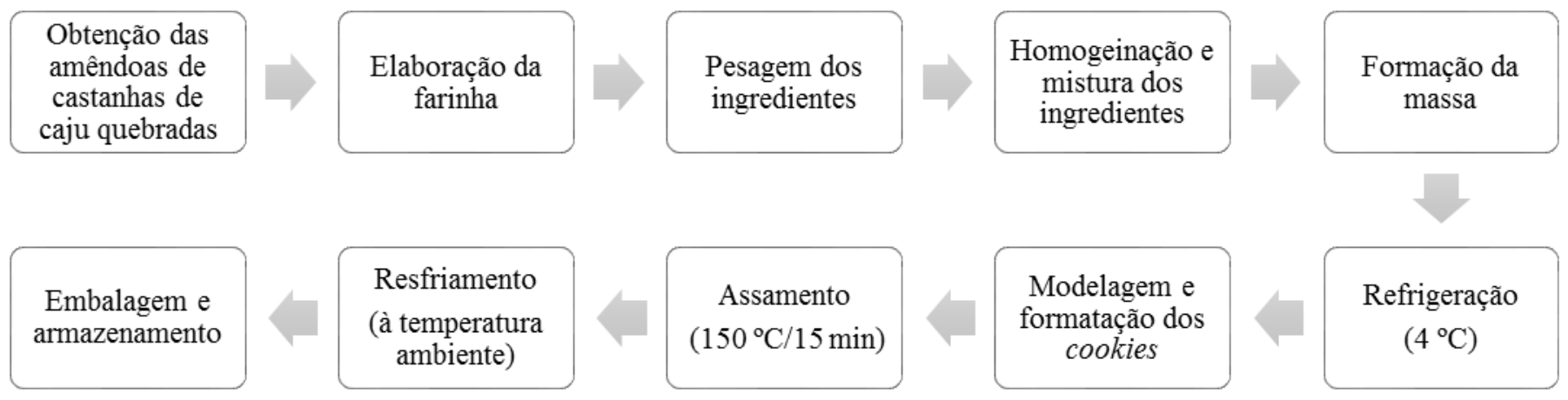

Fonte: Autores (2020).

\subsection{Análises microbiológicas}

As análises microbiológicas foram realizadas segundo as metodologias da American Public Health Association (2001) e de Silva et al. (2017). A Resolução RDC nº 12 de 02 de janeiro de 2001 da Agência Nacional de Vigilância Sanitária ANVISA, como dito por Brasil (2001), estabelece para esse tipo de produto a contagem de coliformes termotolerantes, Staphylococcus aureus e pesquisa de Salmonella. Realizaram-se ainda as contagens de bolores e leveduras e de aeróbios mesófilos.

\subsection{Composição centesimal}

As análises de composição centesimal realizadas nos cookies foram: umidade (\%, g.100 g $\left.{ }^{-1}\right)$; proteínas $(\%$, g.100 g estimada com base no teor total de nitrogênio, determinado pelo método de Micro-Kjeldahl, usando fator de conversão de 6,38; cinzas (\%, g.100 g $\left.\mathrm{g}^{-1}\right)$, de acordo com os métodos descrito pelo Instituto Adolfo Lutz (2008). A determinação quantitativa de lipídios totais (\%, g.100 $\left.\mathrm{g}^{-1}\right)$, foi realizado por extração em Soxhlet, utilizando hexano como solvente (IAL, 2008). A concentração de carboidrato total $\left(\%, \mathrm{~g} .100 \mathrm{~g}^{-1}\right)$ foi calculada por diferença (TBCA-USP, 2020). Todas as análises foram 
realizadas em triplicata. A fibra bruta (FB) foi analisada pela técnica AOCS (Firestone, 2009) do saco de filtro Ankom, onde ocorre a digestão ácida e alcalina da amostra seca e desengordurada durante 30 minutos em cada digestão.

\subsection{Avaliação sensorial}

A pesquisa foi aprovada (Parecer n. 3.314.772) pelo Comitê de Ética em Pesquisa do Instituto Federal de Educação, Ciência e Tecnologia do Ceará - IFCE, respeitando a Resolução n466/2012 do Ministério da Saúde, referente a pesquisas envolvendo seres humanos (Brasil, 2013).

Os participantes receberam e assinaram o Termo de Consentimento Livre e Esclarecido (TCLE). Foram incluídos na análise sensorial indivíduos que aceitaram participar e que não eram alérgicos aos ingredientes utilizados na elaboração dos cookies; foram excluídos os indivíduos que tinham alergia aos ingredientes utilizados.

Os testes sensoriais seguiram a metodologia de Dutcosky (2013), realizados com 120 avaliadores não treinados aptos a participarem. Foram avaliados os atributos: impressão global, aparência, odor, textura e sabor por meio da escala hedônica estruturada de 9 pontos, sendo: 1 - desgostei muitíssimo e 9 - gostei muitíssimo e para intenção de compra, escala estruturada de 5 pontos, sendo: 5 - certamente compraria e 1 - certamente não compraria. Com base nas pontuações por escore obtidas, o índice de aceitabilidade (IA) foi calculado por meio de equação, sendo IA= (Pontuação média obtida para o produto/Maior pontuação atribuída ao produto) x 100 .

\subsection{Análise Estatística}

Os resultados obtidos foram submetidos à análise estatística de variância (ANOVA) e para o teste de comparação de médias foi realizado o teste de Tukey, ao nível de significância de 5\% (p > 0,05), utilizando o programa estatístico Statsoft (2011) de versão 10.0.

\section{Resultados e Discussão}

\subsection{Avaliação microbiológica}

Os resultados das análises microbiológicas das três formulações elaboradas demonstraram ausência para contagem de coliformes termotolerantes, pesquisa de Salmonella sp. em 25 gramas de amostra, contagem de Staphylococcus aureus, bolores e leveduras e aeróbios mesófilos (Tabela 2). Estes resultados asseguram a qualidade higiênico-sanitária na elaboração dos cookies controle e com adição da FCC, confirmando que se encontram aptos para o consumo, de acordo com a legislação vigente (Brasil, 2001).

Tabela 2. Resultados das análises microbiológicas dos cookies com farinha de castanha de caju.

\begin{tabular}{|c|c|c|c|c|c|}
\hline Formulação & & $\overline{\text { Aná }}$ & microbiológicas & & \\
\hline & $\begin{array}{c}\text { Coliformes } \\
\text { Termotolerantes } \\
\text { (NMP/g) }\end{array}$ & Salmonella sp. & $\begin{array}{c}\text { Staphylococcus } \\
\text { aureus } \\
\text { (UFC/g) }\end{array}$ & $\begin{array}{c}\text { Bolores e } \\
\text { leveduras } \\
\text { (UFC/g) }\end{array}$ & $\begin{array}{c}\text { Aeróbios } \\
\text { mesófilos } \\
\text { (UFC/g) }\end{array}$ \\
\hline CO & $<3$ & Ausência & Ausência & Ausência & Ausência \\
\hline C50 & $<3$ & Ausência & Ausência & Ausência & Ausência \\
\hline C75 & $<3$ & Ausência & Ausência & Ausência & Ausência \\
\hline Legislação* & $10^{2}$ & Ausência em $25 \mathrm{~g}$ & $5 \times 10^{3}$ & - & - \\
\hline
\end{tabular}

C0: controle; C50: 50\% de FCC; C75: 75\% de FCC. *Brasil (2001). Fonte: Autores (2020). 
Sabino, Oliveira, Feitosa, Feitosa e Oliveira (2017), ao realizarem análises microbiológicas em suas formulações de biscoitos tipo cookie, utilizando a farinha do resíduo agroindustrial do caju, obtiveram resultados semelhantes ao do presente trabalho, com baixas contagens de bolores e leveduras e de coliformes totais, bem como ausência de coliformes termotolerantes, estando dentro do exigido pela legislação.

Melo et al. (2017), ao elaborarem biscoitos com a adição de farinha da castanha de caju, testando diferentes adoçantes, avaliaram também suas características microbiológicas, atestando a ausência na contagem de coliformes termotolerantes e de bolores e leveduras em todas as formulações. Zuniga, Coelho, Ferreira, Resende e Almeida (2011), ao avaliarem a vida de prateleira de biscoito integral de castanha de caju por 80 dias, observaram variação apenas para a contagem de bolores e leveduras durante a estocagem; com ausência de coliformes termotolerantes e Salmonella em todo o período.

Vale ressaltar que esses resultados satisfatórios do ponto de vista microbiológico podem estar relacionados ao baixo teor de água presente nos cookies elaborados (Tabela 3). Segundo Dias, Santana, Pinto e Oliveira (2016), baixos percentuais de umidade são ideais para obter-se um produto com vida de prateleira estendida, pois não favorecem o crescimento microbiano e as alterações bioquímicas e sensoriais, retardando sua deterioração.

\subsection{Caracterização centesimal}

Os dados da caracterização centesimal das três formulações dos cookies elaboradas estão descritos na Tabela 3.

Tabela 3. Composição centesimal aproximada dos cookies com a FCC.

\begin{tabular}{lcccccc}
\hline Formulação & \multicolumn{5}{c}{ Composição Centesimal Aproximada $\left(\mathbf{g} / \mathbf{1 0 0} \mathbf{~ g}^{\mathbf{- 1}} \pm\right.$ desvio padrão) } \\
\hline & Umidade & Lipídeos & Proteína total & Fibra bruta & Cinzas & Carboidrato total \\
\hline C0 & $5,35 \pm 0,07 \mathrm{c}$ & $24,34 \pm 0,89 \mathrm{a}$ & $11,40 \pm 0,28 \mathrm{a}$ & $0,88 \pm 0,00 \mathrm{~b}$ & $1,63 \pm 0,01 \mathrm{a}$ & $56,39 \pm 1,10 \mathrm{a}$ \\
C50 & $6,40 \pm 0,14 \mathrm{~b}$ & $18,44 \pm 0,48 \mathrm{~b}$ & $13,56 \pm 1,14 \mathrm{a}$ & $2,79 \pm 0,12 \mathrm{a}$ & $2,31 \pm 0,30 \mathrm{a}$ & $56,49 \pm 0,62 \mathrm{a}$ \\
C75 & $11,75 \pm 0,07 \mathrm{a}$ & $19,05 \pm 0,90 \mathrm{~b}$ & $13,22 \pm 0,71 \mathrm{a}$ & $2,81 \pm 0,05 \mathrm{a}$ & $1,94 \pm 0,02 \mathrm{a}$ & $51,23 \pm 0,35 \mathrm{~b}$ \\
\hline
\end{tabular}

C0: controle; C50: 50\% de FCC; C75: 75\% de FCC. Médias seguidas pela mesma letra na coluna não apresenta diferença significativa (p $\geq$ 0,05). Fonte: Autores (2020).

Observou-se diferença significativa entre as três formulações para o teor de umidade $(\mathrm{p}<0,05)$, que aumentou à medida que a FCC foi acrescentada. Situação similar foi observada no estudo de Dias et al. (2016), que elaboraram cookies com farinha de aveia: o aumento da quantidade da farinha proporcionou o aumento no teor de umidade. Sabino et al. (2017), em seu estudo já citado e Medeiros, Santos, Ferreira, Viana e Machado (2020), no desenvolvimento de biscoitos a partir do resíduo da extração de suco de caju do cerrado Goiano, obtiveram teores de umidade superiores aos encontrados neste trabalho, ressaltando que quanto menor o teor de umidade, maior é a vida de prateleira do produto.

Para o teor de lipídios, observou-se diferença significativa entre a formulação controle e as amostras com FCC ( $\mathrm{p}<$ 0,05), onde a maior quantidade lipídica foi observada na formulação C0. Medeiros et al. (2020), obtiveram teores inferiores em seu trabalho (de 7,40 g/100 g $\mathrm{g}^{-1}$ ). Melo et al. (2017), observaram teores superiores aos do estudo em questão, variando de 28 a $34 \mathrm{~g} / 100 \mathrm{~g}^{-1}$, com diferenças significativas entre todas as formulações, distintas pelo tipo de adoçante utilizado. Os autores salientam que em estudo do Instituto Nacional de Metrologia, Qualidade e Tecnologia, a castanha de caju apresenta o menor teor lipídico dentre as frutas oleaginosas mais consumidas no Brasil (BRASIL, 2012).

$\mathrm{O}$ teor proteico não foi significativamente diferente entre as três formulações ( $\mathrm{p} \geq 0,05)$, variando de 11,40 a 13,56 $\mathrm{g} / 100 \mathrm{~g}^{-1}$, onde a maior quantidade foi identificada na amostra C50. Esses resultados foram superiores aos encontrados por Melo et al. (2017), e Sabino et al. (2017), e Medeiros et al. (2020), em seus estudos. Porém, a Instrução Normativa $\mathrm{n}^{\circ} 75$ 
(Brasil, 2020), considera que o alimento é "fonte de proteínas" quando possui no mínimo $5 \mathrm{~g}$ de proteínas por porção $(30 \mathrm{~g})$. Diante disso, os cookies elaborados não podem ser considerados alimentos "fonte de proteínas", apresentando 3,42 g/30 g, 4,07 $\mathrm{g} / 30 \mathrm{~g}$ e 3,97 g/30 g de proteínas para C0, C50 e C75, respectivamente.

$\mathrm{Na}$ determinação de fibra bruta, observa-se diferença estatística significativa entre a formulação $\mathrm{C} 0$ e as formulações com FCC (p < 0,05). Obteve-se um teor de $0,88 \mathrm{~g} / 100 \mathrm{~g}^{-1}$ para a formulação controle, e à medida que se acrescentou a FCC, o teor de fibra aumentou no cookie, chegando a $2,81 \mathrm{~g} / 100 \mathrm{~g}^{-1}$ para a C75. Matias, Oliveira, Gertrudes e Magalhães (2005), ao analisar o teor de fibra no bagaço desidratado do caju, encontrou valor elevado, de 41,53 g/100 g g $^{-1}$ Morais et al. (2018), ao elaborarem cupcake adicionado de farinha de fibra de caju obtiveram teores semelhantes ao do estudo em questão, variando de 0,49 a 2,78 g/100 g $\mathrm{g}^{-1}$. Já Owiredu et al. (2014), em seu estudo realizado em Kumasi, cidade do Gana, não encontraram teor de fibra em nenhuma das formulações, seja na amostra controle ou com substituições de farinha de trigo por farinha de castanha de caju, podendo implicar nas variações que diferentes regiões de cultivo de um mesmo fruto podem ocasionar no alimento.

A IN n 75 (Brasil, 2020), considera que o alimento é "fonte de fibras alimentares" quando possui no mínimo 2,5 g de fibras por porção $(30 \mathrm{~g})$, contendo os cookies elaborados uma concentração inferior, de $0,27 \mathrm{~g} / 30 \mathrm{~g}, 0,84 \mathrm{~g} / 30 \mathrm{~g}$ e $0,84 \mathrm{~g} / 30 \mathrm{~g}$ de fibras alimentares para $\mathrm{C} 0, \mathrm{C} 50$ e $\mathrm{C} 75$, respectivamente.

Os teores de cinzas foram significativamente iguais entre as três formulações elaboradas ( $p \geq 0,05)$, inferindo que a adição de FCC não exerceu influência na composição mineral dos cookies. O mesmo resultado foi encontrado por Morais et al. (2018), em seu estudo, onde não houve diferença significativa entre as formulações, e o teor de cinzas foi maior na amostra que continha menor adição de farinha de fibra de caju.

$\mathrm{O}$ teor de carboidratos foi obtido por diferença, observando-se que a amostra C75 diferiu das outras duas formulações ( $p>0,05$ ), obtendo teor inferior das demais. Os teores encontrados por Silva, Andrade, Silva e Gomes (2019), e Medeiros et al. (2020), foram um pouco superiores ao desse estudo, estando diretamente relacionado a diferente composição centesimal de cada produto elaborado.

\subsection{Avaliação sensorial}

Participaram da análise sensorial 83 avaliadores do sexo feminino e 37 do sexo masculino. Quanto ao teste de aceitação por escala hedônica, os cookies obtiveram uma boa aceitação, com escores médios compreendidos entre 6,51 e 7,30, correspondendo a gostei ligeiramente e gostei moderadamente (Tabela 4).

Tabela 4. Médias dos escores de aceitação sensorial dos cookies com farinha de castanha de caju.

\begin{tabular}{lccccc}
\hline \multirow{2}{*}{ Formulação } & \multicolumn{5}{c}{ Aceitação (escore médio \pm desvio padrão) } \\
\hline & Impressão global & Aparência & Odor & Textura & Sabor \\
\hline C0 & & & & \\
C50 & $6,83 \pm 1,60 \mathrm{a}$ & $6,87 \pm 1,75 \mathrm{a}$ & $6,78 \pm 1,53 \mathrm{a}$ & $6,51 \pm 1,90 \mathrm{~b}$ & $6,56 \pm 1,86 \mathrm{~b}$ \\
C75 & $7,01 \pm 1,59 \mathrm{a}$ & $6,85 \pm 1,55 \mathrm{a}$ & $7,00 \pm 1,43 \mathrm{a}$ & $7,25 \pm 1,52 \mathrm{a}$ & $7,23 \pm 1,71 \mathrm{a}$ \\
\hline
\end{tabular}

C0: controle; C50: 50\% de FCC; C75: 75\% de FCC. Médias seguidas pela mesma letra na coluna não apresenta diferença significativa (p $\geq$ 0,05). Fonte: Autores (2020).

Para os atributos impressão global, aparência e odor não se observou diferença significativa $(p \geq 0,05)$ entre as três formulações elaboradas. Já para os atributos textura e sabor houve diferença significativa $(p<0,05)$ entre a amostra $\mathrm{C} 0$ e as amostras C50 e C75 que possuem FCC em sua composição, onde essas não diferiram entre si. Isso indica que, para os 
avaliadores, a concentração de farinha utilizada não possibilitou diferenciar as características de textura e sabor entre C50 e C75, contudo, foi possível observar que existia diferença entre a amostra que não continha FCC das que continham.

Os analisadores apontaram que o cookie sem a FCC estava com a textura muito diferente dos demais, e que os biscoitos com FCC apresentavam um aspecto menos "crocante".

Esses resultados são satisfatórios, visto que, as maiores médias, variando de 7,15 a 7,30 foram para a formulação que possuía $75 \%$ da farinha de castanha e apenas $25 \%$ da farinha de trigo integral, sendo considerada a formulação mais aceita pelos consumidores. No estudo de Ojinnaka e Agubolum (2013), observou-se que a formulação com maior aceitação possuía $70 \%$ de farinha de trigo e 30\% de farinha de castanha de caju em sua composição, praticamente o oposto do encontrado no presente trabalho. Diante disso, pode-se perceber que as diferentes culturas de cada região exercem influência nas diferentes escolhas, preferências ou seleções para um mesmo tipo de produto ou modificação realizada em sua formulação.

Sabino et al. (2017), em seu estudo utilizando a farinha do resíduo de caju branqueada, não observaram diferença significativa em nenhum dos atributos dos biscoitos elaborados, mas ainda assim, o biscoito com farinha do resíduo de caju branqueada obteve escores médios maiores que a não branqueada. Já Silva et al. (2019), ao produzirem biscoitos utilizando a farinha do caroço do abacate, obtiveram escores médios semelhantes ao do presente estudo, com exceção do atributo sabor, onde a média hedônica foi 4,6 (“desgostei ligeiramente”), estando associado ao sabor residual amargo da farinha.

Quanto a aceitabilidade, os resultados indicaram que todos os cookies elaborados foram aprovados pelos consumidores, onde para todos os atributos, as três formulações obtiveram IA acima de 70\% (Figura 2), concordando com Dutcosky (2013), ao afirmar que para um produto ser considerado aceitável, ele precisa apresentar no mínimo $70 \%$ de aceitabilidade.

Figura 2. Índice de Aceitabilidade dos cookies com farinha de castanha de caju.

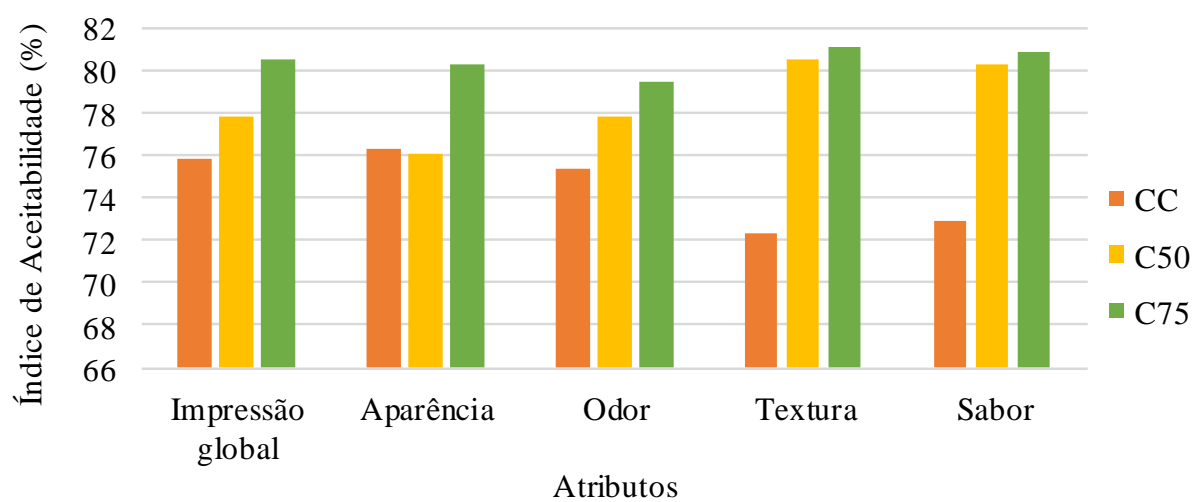

CC: controle; C50: 50\% de FCC; C75: 75\% de FCC. Fonte: Autores (2020).

Os maiores percentuais obtidos foram para a formulação $\mathrm{C} 75$, com IA em torno de $80 \%$ para todos os atributos analisados. Esses resultados são satisfatórios, pois indicam que a maior adição de FCC agradou os consumidores que participaram do teste, influenciando na aceitabilidade do produto. Oliveira et al. (2020), observaram a mesma situação em sua pesquisa, onde a amostra com a maior proporção de substituição por farinha de banana verde destacou-se com maior IA. Sabino et al. (2017), também obtiveram percentuais de IA variando de $70 \%$ a $80 \%$ em seu trabalho.

Para intenção de compra, 80,9\% das respostas dos avaliadores situaram-se entre os escores 4 e 5, indicando que provavelmente comprariam ou certamente comprariam o cookie elaborado. Nos estudos de Silva et al. (2019), e Oliveira et al. 
(2020), os resultados foram similares, onde os avaliadores expressaram atitude positiva de compra caso os cookies elaborados estivessem disponíveis no mercado varejista.

\section{Conclusão}

Os resultados obtidos neste trabalho revelam a qualidade higiênico-sanitária na elaboração dos biscoitos produzidos, demonstrando que se encontravam aptos para o consumo. Todos os cookies elaborados foram aprovados pelos consumidores, com destaque para a formulação C75, com $75 \%$ da FCC, que apresentou índice de aceitabilidade em torno de $80 \%$ para todos os atributos analisados e alto percentual de intenção de compra.

Conclui-se assim, que os biscoitos elaborados utilizando a farinha da castanha de caju constituem um produto com potencial de mercado, representando uma alternativa viável de aproveitamento das amêndoas quebradas, agregando valor aos produtos, além de apresentarem importantes características nutricionais.

Como sugestão para abordagens futuras, recomenda-se a avaliação do custo-benefício de cada formulação elaborada, com propósito de conhecer o custo unitário final dos cookies, disseminando as informações para a academia e para a indústria alimentícia, viabilizando o processo de elaboração, comercialização e consumo destes produtos.

\section{Agradecimentos}

Ao CNPq pelo financiamento da bolsa PIBITI concedida e ao Instituto Federal do Ceará pelo apoio na realização da pesquisa.

\section{Referências}

American Public Health Association (2001). Compendium of Methods for the Microbiological Examination of Foods. (4th ed.) 676 p.

Chaves D. C., Azevedo S. S., \& Oliveira M. A. (2020) Elaboração de biscoito tipo cookies adicionado de castanha de caju (Anacardium sp). Extensão Rural em Foco Apoio à Agricultura Familiar, Empreendedorismo e Inovação, cap. 4., (1 ${ }^{\mathrm{a}}$ ed.,) v. 2. https://doi.org/10.37885/200600443

Companhia Nacional de Abastecimento (2020). Análise mensal de castanha de caju. https://www.conab.gov.br/info-agro/analises-do-mercado-agropecuarioe-extrativista/analises-do-mercado/historico-mensal-de-castanha-de-caju.

Companhia Nacional de Abastecimento (2017). Castanha de caju: panorama da cajucultura no Ceará. CONAB: Fortaleza- CE. http://www.conab.gov.br/OlalaCMS/uploads/arquivos/17_02_09_14_35_39_cajucultura_no_ceara___2017.pdf.

Dias, B. F., Santana, G. S., Pinto, E. G., \& de Oliveira, C. F. D. (2016). Caracterização físico-química e análise microbiológica de cookie de farinha de aveia. Journal of Neotropical Agriculture, 3(3), 10-14.

Dutcosky, S. D. (2013). Análise sensorial de alimentos. (4⿳a ed.) rev. ampl. Curitiba: Champagnat, 531p.

Firestone, D. (2009). Official methods and recommended practices of the AOCS. AOCS, 2009.

Freitas, J. B., \& Naves, M. M. V. (2010). Composição química de nozes e sementes comestíveis e sua relação com a nutrição e saúde. Revista de Nutrição, 23(2), 269-279.

Gadani, B. C., Mileski, K. M., Peixoto, L. S., \& Agostini, J. S. (2017). Características físicas e químicas da farinha de castanha de caju armazenada e embalada em diferentes embalagens. Food Science and Technology, 37(4),657-662. https://doi.org/10.1590/1678-457x.27516

Ibrahim, T. A., Omosolis, V., Oloye, D. A., Aladekoyi, G., \& Ogundowole, O. (2010). Functional properties of roasted and defatted cashew nut flour. Electronic Journal of Environmental Agricultural and Food Chemistry, 10(2), 2135-2138.

Instituto Adolfo Lutz (2008). Métodos físico-químicos para análise de alimentos: Instituto Adolfo Lutz, 1020 p.

Instituto Nacional de Metrologia, Normalização e Qualidade Industrial. Relatório sobre análise de teor de gordura e fitosteróis em nuts (amêndoa, amendoim, avelã, castanha do Pará, macadâmia e nozes. <http://www.inmetro.gov.br/consumidor/produtos/nuts.pdf>

Instituto Brasileiro de Geografia e Estatística (2017). Levantamento sistemático da produção agrícola. ftp://ftp.ibge.gov.br/Producao_Agricola/Levantamento_Sistematico_da_Producao_Agricola[mensal]/Fasciculo/lspa_201701.pdf.

Kauark, F. D. S., Manhães, F. C., \& Medeiros, C. H. (2010). Metodologia da pesquisa: guia prático. (1 ed.). Itabuna: Via Litterarum, 88 p. 
Lima, J. R., Pinto, G. A. S., \& Magalhães, H. C. R. (2018). Óleo da amêndoa de castanha-de-caju: métodos de extração. Embrapa Agroindústria TropicalBoletim de Pesquisa e Desenvolvimento (INFOTECA-E).

Matias, M. F. O., Oliveira, E. L., Gertrudes, E., \& Magalhães, M. M. A. (2005). Use of fibres obtained from cashew (Anacardium occidentale, L) and guava (Psidium guajava) fruits for enrichment of food products. Brazilian Archives of Biology and Technology, 48(spe), 143-150. https://doi.org/10.1590/S151689132005000400018

Medeiros, J. S., Santos, L. S. dos, Ferreira, S. V., Viana, L. F., \& Machado, A. R. (2020). Development of biscuits from the residue from the extraction of cashew juice from the Cerrado of Goiás. Research, Society and Development, 9(7), e39973082. http://dx.doi.org/10.33448/rsd-v9i7.3082

Melo, A. B. P., Oliveira, E. N. A., Feitosa, B. F., Feitosa, R. M., \& Oliveira, S. N. (2017). Elaboração e caracterização de biscoitos adicionados de farinha de castanha de caju com diferentes adoçantes. Revista Brasileira de Agrotecnologia, 7(2), 145-150.

Ministério da Saúde. Agência Nacional de Vigilância Sanitária - ANVISA. Instrução Normativa $n^{\circ} 75$, de 08 de outubro de 2020, estabelece os requisitos técnicos para declaração da rotulagem nutricional nos alimentos embalados. Diário Oficial [da] União, Brasília, 09 out. 2020. Edição 195, Seção 1, p. 113.

Ministério da Saúde. Agência Nacional de Vigilância Sanitária - ANVISA. Resolução RDC no 12, de 02 de janeiro de 2001, aprova o Regulamento Técnico sobre padrões microbiológicos para alimentos. Diário Oficial [da] União, Brasília, 10 jan. 2001. Edição 07.

Ministério da Saúde. Conselho Nacional de Saúde. Resolução no 466 de 12 de dezembro de 2012, aprova diretrizes e normas regulamentadoras de pesquisas envolvendo seres humanos. Diário Oficial [da] União, Brasília, 13 jun. 2013. Edição 12, Seção 1, p. 59.

Morais, E. C. de., Patias, S. G. O., Costa, E. S., Sandri, D. D. O., Picanço, N. F. M., \& de Faria, R. A. P. G. (2018). Elaboração de cupcake adicionado de farinha de fibra de caju: caracterização físico-química e sensorial. REBRAPA-Brazilian Journal of Food Research, 9(2), 1-14. https://doi.org/10.3895/rebrapa.v9n2.5537

Ojinnaka, M. C., \& Agubolum, F. U. (2013). Nutritional and sensory properties of cashew nut-wheat based cookies. American Journal of Food and Nutrition, 3(3), 127-134. https://doi.org/10.5251/ajfn.2013.3.3.127.134

Oliveira, P. V. C. de, Queiroz, B. C. A., Piovesan, N., de Oliveira, P. V. C., de Carvalho Lima, M. H., Pereira, A. S., \& de Oliveira Lima, W. (2020). Substituição da farinha de trigo por farinha de banana verde na elaboração de biscoito tipo cookie. Brazilian Journal of Development, 6(10), 75662-75672. https://doi.org/10.34117/bjdv6n10-117

Owiredu, I., Laryea, D., \& Barimah, J. (2014). Evaluation of cashew nut flour in the production of biscuit. Nutrition \& Food Science, 44(3), 204211. https://doi.org/10.1108/NFS-06-2013-0067

Paiva, F. F. A., Neto, R. M. S., Pessoa, P. F. A. P., \& Leite, L. A. S. (2006). Processamento de castanha de caju. Brasília, DF: Embrapa Informação Tecnológica, ISBN 85-7383-359-9, 2006.

Sabino, V. G., Oliveira, E. N. A., Feitosa, B. F., Feitosa, R. M., \& Oliveira, S. N. (2017). Desenvolvimento e caracterização de biscoitos tipo cookie de farinha do resíduo agroindustrial do caju. Revista Brasileira de Agrotecnologia, 7(2), 38-44.

Silva, I. G. D., Andrade, A. P. C. D., Silva, L. M. R. D., \& Gomes, D. S. (2019). Elaboração e análise sensorial de biscoito tipo cookie feito a partir da farinha do caroço de abacate. Brazilian Journal of Food Technology, 22. e2018209, 1-10. https://doi.org/10.1590/1981-6723.20918

Silva, N., Junqueira, V. C. A., Silveira, N. F. A., Taniwaki, M. H., Gomes, R. A. R., \& Okazaki, M. M. (2017). Manual de métodos de análise microbiológica de alimentos e água. (5a ed.), Blucher.

Statsoft. (2011). Statistica for Windows - Computer program manual, version 10.0. Tulsa: Statsoft Inc.

Tabela Brasileira de Composição de Alimentos (TBCA) (2020). Universidade de São Paulo (USP). Food Research Center (FoRC). Versão 7.1. São Paulo. http://www.fcf.usp.br/tbca.

Zuniga, A. D. G., Coelho, A. F. S., Ferreira, E., Resende, E. A., \& Almeida, K. D. (2011). Avaliação da vida de prateleira de biscoito de castanha de caju tipo integral. Revista Brasileira de Produtos Agroindustriais, 13(3), 251-256. 